Proyecciones Journal of Mathematics

Vol. 28, No 3, pp. 203-207, December 2009.

Universidad Católica del Norte

Antofagasta - Chile

\title{
A SIMPLE NATURAL APPROACH TO THE UNIFORM BOUNDEDNESS PRINCIPLE FOR MUTILINEAR MAPPINGS
}

\author{
A. THIAGO BERNARDINO \\ UNIVERSIDADE FEDERAL DE PERNAMBUCO, BRASIL \\ Received: February 2009. Accepted : July 2009
}

\begin{abstract}
The goal of this note is to give a new, simple and elegant proof to the Uniform Boundedness Principle (UBP) to m-linear mappings, which surprisingly, as far as we know, does not appear in the literature. The multilinear UBP is well-known for specialists but the original proof (presented in [4]) seems a little bit unnatural and uses the linear UBP. In the present note we show a quite simple argument which does not need to invoke the linear UBP and, when $m=1$, recovers the classical proof of the linear case. As an immediate consequence, we obtain the Banach-Steinhaus Theorem (BST) for multilinear mappings.
\end{abstract}




\section{Introduction}

The theory of multilinear mappings and polynomials between Banach spaces is a natural extension of linear functional analysis and has been developed through different directions. In this context, it is natural to study whether versions of classical linear results hold in the multilinear setting (see, e.g., $[2])$.

The multilinear versions of the Uniform Boundedness Principle (UBP) and Banach-Steinhaus Theorem (BST) are well-known for specialists, but we think that the proofs presented in the literature are not the most natural ones. The UBP for multilinear maps is proved in [4] and the BST for multilinear maps is a folkloric result and the standard proof is an adaptation of [1, Ex 1.11]. Both proofs need to use the linear UBP. Precisely, in [4] the proof of the multilinear UBP seems unnecessarily complicated and invokes twice the linear version of the UBP and uses an induction argument; the proof of the multilinear BST uses that every separately continuous $m$-linear map (defined in Banach spaces) is continuous and this is a consequence of the linear UBP (see [1, Theorem 1.2]).

The main goal of this short note is to present easy proofs of the multilinear UBT and BST without using the linear UBT. Besides, our proofs follow the same lines of the linear respective proofs. In resume, we know that the subject of this note (a multilinear UBT and BST) is well-known, but we think that our presentation contributes to redeem a hidden feature of the proof of the linear UBT: it is naturally and elegantly adaptable to multilinear mappings. Our approach follows the same line of the proof of the Banach-Steinhaus Theorem for homogeneous polynomials in [3, Theorem 2.6]. However, the proof of the multilinear case is technically different from the proof of the polynomial case, since there is no polarization formula for non-symmetric multilinear mappings.

From now on $m$ is a positive integer, $E_{i}, i=1, \ldots, m$, and $F$ are normed linear spaces over the real or complex scalar fields. If $x \in E$ and $r>0$, $B_{E}(x ; r)$ denotes the closed ball of center $x$ and radius $r$. It is well known that $E_{1} \times \cdots \times E_{m}$, with $E_{1}, \ldots, E_{m}$ Banach spaces, is a Banach space with the norm $\left\|\left(x_{1}, \ldots, x_{m}\right)\right\|=\max _{1 \leq i \leq m}\left\|x_{i}\right\|$.

The space of continuous $m$-linear mappings from $E_{1} \times \cdots \times E_{m}$ to $F$ is denoted by $L\left(E_{1}, \ldots, E_{m} ; F\right)$. It is easy to prove that an $m$-linear map $A: E_{1} \times \cdots \times E_{m} \rightarrow F$ is continuous if, and only if,

$$
\|A\|:=\sup _{\left\|x_{i}\right\| \leq 1, i=1, \ldots, m}\left\|A\left(x_{1}, \ldots, x_{m}\right)\right\|<\infty .
$$




\section{Uniform Boundedness and Banach-Steinhaus Theorem for multilinear mappings}

The reader will note that the proof of the next theorem (UBP for multilinear mappings) is inspired on the classical proof of the linear case but, as we have mentioned, contrary to the proof in [4], our proof does not require invoking the linear UBP. If we take $m=1$, our proof is precisely the proof of the linear UBP.

Theorem 1 (Uniform Boundedness Principle for multilinear mappings). Let $E_{1}, \ldots, E_{m}$ be Banach spaces, $F$ be a normed space and $\left\{T_{i}\right\}_{i \in I}$ be a family in $L\left(E_{1}, \ldots, E_{m} ; F\right)$. If

$$
\sup _{i \in I}\left\|T_{i}\left(x_{1}, \ldots, x_{m}\right)\right\|<\infty \text { for all }\left(x_{1}, \ldots, x_{m}\right) \in E_{1} \times \cdots \times E_{m}
$$

then

$$
\sup _{i \in I}\left\|T_{i}\right\|<\infty
$$

Proof. For each positive integer $n$, let

$$
A_{n}=\left\{\left(x_{1}, \ldots, x_{m}\right) \in E_{1} \times \cdots \times E_{m} ; \sup _{i \in I}\left\|T_{i}\left(x_{1}, \ldots, x_{m}\right)\right\| \leq n\right\} .
$$

Note that each $A_{n}$ is a closed subset of $E_{1} \times \cdots \times E_{m} \stackrel{(2.1)}{=} \bigcup_{n=1}^{\infty} A_{n}$. From the Baire Category Theorem we know that there is a positive integer $n_{0}$ such that $A_{n_{0}}$ has non-empty interior. Let $\left(a_{1}, \ldots, a_{m}\right)$ be an interior point of $A_{n_{0}}$. So, there is a constant $r>0$ so that $B_{E_{1} \times \cdots \times E_{m}}\left(\left(a_{1}, \ldots, a_{m}\right) ; r\right)$ is contained in $A_{n_{0}}$.

Let $i \in I$. We have $\left\|T_{i}\left(y_{1}, a_{2}+y_{2}, \ldots, a_{m}+y_{m}\right)+T_{i}\left(a_{1}, a_{2}+y_{2}, \ldots, a_{m}+y_{m}\right)\right\|$ $=\left\|T_{i}\left(a_{1}+y_{1}, \ldots, a_{m}+y_{m}\right)\right\| \leq n_{0}$, for every $\left(y_{1}, \ldots, y_{m}\right) \in B_{E_{1} \times \cdots \times E_{m}}((0, \ldots, 0) ; r)$. Hence

$$
\left\|T_{i}\left(y_{1}, a_{2}+y_{2}, \ldots, a_{m}+y_{m}\right)\right\| \leq n_{0}+\left\|T_{i}\left(a_{1}, a_{2}+y_{2}, \ldots, a_{m}+y_{m}\right)\right\| \leq 2 n_{0}
$$

for every $\left(y_{1}, \ldots, y_{m}\right) \in B_{E_{1} \times \cdots \times E_{m}}((0, \ldots, 0) ; r)$.

Using the same argument, for every $\left(z_{1}, \ldots, z_{m}\right) \in B_{E_{1} \times \cdots \times E_{m}}((0, \ldots, 0) ; r)$, we get $\left\|T_{i}\left(z_{1}, z_{2}, a_{3}+z_{3}, \ldots, a_{m}+z_{m}\right)+T_{i}\left(z_{1}, a_{2}, a_{3}+z_{3}, \ldots, a_{m}+z_{m}\right)\right\|$

$=\left\|T_{i}\left(z_{1}, a_{2}+z_{2}, \ldots, a_{m}+z_{m}\right)\right\| \stackrel{(2.2)}{\leq} 2 n_{0}$,

and 


$$
\left\|T_{i}\left(z_{1}, z_{2}, a_{3}+z_{3}, \ldots, a_{m}+z_{m}\right)\right\| \stackrel{(2.3)}{\text { and }(2.2)} \leq 2 n_{0}+2 n_{0}=4 n_{0} .
$$

By repeating this argument $m$ times, we have

$$
\left\|T_{i}(x)\right\| \leq 2^{m} n_{0}
$$

for all $x \in B_{E_{1} \times \cdots \times E_{m}}((0, \ldots, 0) ; r)$. Since $i \in I$ is arbitrary, we easily get

$$
\sup _{i \in I}\left\|T_{i}\right\| \leq \frac{2^{m} n_{0}}{r^{m}}
$$

The following corollary is an immediate consequence of Theorem 1 :

Corollary 1 (Banach-Steinhaus Theorem for multilinear mappings). Let $E_{1}, \ldots, E_{m}$ be Banach spaces, $F$ be a normed space and $\left(A_{n}\right)_{n=1}^{\infty}$ be a sequence in $L\left(E_{1}, \ldots, E_{m} ; F\right)$ so that for each $x_{j} \in E_{j}$, the sequence $\left(A_{n}\left(x_{1}, \ldots, x_{m}\right)\right)_{n=1}^{\infty}$ is convergent. If $A\left(x_{1}, \ldots, x_{m}\right)=\lim _{n \rightarrow \infty} A_{n}\left(x_{1}, \ldots, x_{m}\right)$ for each $x_{j} \in E_{j}$, then $A \in L\left(E_{1}, \ldots, E_{m} ; F\right)$.

Proof. It is clear that $A$ is $m$-linear. Since $\left(A_{n}\left(x_{1}, \ldots, x_{m}\right)\right)_{n=1}^{\infty}$ is convergent, it follows that

$$
\sup _{n}\left\|A_{n}\left(x_{1}, \ldots, x_{m}\right)\right\|<\infty \text { for every }\left(x_{1}, \ldots, x_{m}\right) \in E_{1} \times \cdots \times E_{m} .
$$

From the previous result, we conclude that there is a real number $C>0$ such that

$$
\sup _{n}\left\|A_{n}\right\|<C
$$

So,

$$
\left\|A_{n}\left(x_{1}, \ldots, x_{m}\right)\right\| \leq\left\|A_{n}\right\|\left\|x_{1}\right\| \cdots\left\|x_{m}\right\|<C\left\|x_{1}\right\| \cdots\left\|x_{m}\right\|,
$$

and, making $n \rightarrow \infty$, it follows that $A$ is continuous.

Acknowledgement 1. The author thanks Prof. Daniel Pellegrino for introducing him to the subject of this note, and also thanks the referee for his/her helpful suggestions. 


\title{
References
}

[1] A. Defant and K. Floret, Tensor Norms and Operators Ideals, NorthHolland Mathematics Studies, 176, North-Holland, (1993).

[2] C. Fernandez, The closed graph theorem for multilinear mappings, International Journal of Mathematics and Mathematical Sciences, 19, pp. 407-408, (1996).

[3] J. Mujica, Complex Analysis in Banach spaces, North-Holland Mathematics Studies 120, North-Holland, (1986).

[4] I. Sandberg, Multilinear maps and uniform boundedness. IEEE Trans. Circuits and Systems 32, pp. 332-336, (1985).

\author{
A. Thiago Bernardino \\ Departamento de Matemática \\ Universidade Federal de Pernambuco \\ 50.740-540 - Recife, \\ Brazil \\ e-mail : thiagobernardino@yahoo.com.br
}

